Revue d'histoire de l'Amérique française

TEVUE D.HISTOIRE DE L'AMÉRIQUE FRANÇAISE

\title{
Rameau de Saint-Père et le métier d'historien
}

\section{Pierre Trépanier et Lise Trépanier}

Volume 33, numéro 3, décembre 1979

URI : https://id.erudit.org/iderudit/303792ar

DOI : https://doi.org/10.7202/303792ar

Aller au sommaire du numéro

Éditeur(s)

Institut d'histoire de l'Amérique française

ISSN

0035-2357 (imprimé)

1492-1383 (numérique)

Découvrir la revue

Citer cet article

Trépanier, P. \& Trépanier, L. (1979). Rameau de Saint-Père et le métier d'historien. Revue d'histoire de l'Amérique française, 33(3), 331-355.

https://doi.org/10.7202/303792ar d'utilisation que vous pouvez consulter en ligne.

https://apropos.erudit.org/fr/usagers/politique-dutilisation/ 


\title{
RAMEAU DE SAINT-PẼRE ET LE MÉTIER D'HISTORIEN
}

\author{
PIERRE et LISE TRÉPANIER* \\ Département d'histoire-géographie \\ Université de Moncton
}

En France, le nom de Rameau de Saint-Père n'évoque plus rien. Mais au Québec et, plus encore en Acadie, on ne l'a pas oublié. Parfois on loue l'historien; souvent on instruit son procès en responsabilité morale ${ }^{1}$. Cette survie étonnante, dans nos débats, du vieil érudit, suffirait à attirer sur lui l'attention de ceux qu'intéressent l'historiographie et l'histoire des idéologies. Mais il y a plus. Rameau de Saint-Père est, à notre sens, le fondateur de l'historiographie acadienne de langue française. Pour qui a lu Édouard Richard, Émile Lauvrière, Antoine Bernard et Robert Rumilly, cela ne fait aucun doute. Au regard de l'histoire des idéologies, Rameau a aussi joué un rôle éminent, en Acadie. Nous doutons qu'il soit à l'origine du vieux nationalisme acadien, fondé sur le providentialisme et le traditionalisme, mais il en a assemblé les éléments épars en une doctrine cohérente, il lui a accordé l'aval prestigieux d'un intellectuel de la vieille Europe et il lui a conféré, en quelque sorte, la consécration de l'histoire.

À nos yeux cependant, malgré son intérêt intrinsèque, Rameau s'est présenté comme un moyen d'explorer un peu plus en profondeur les idéologies et l'historiographie en soi et dans leurs

* P. Trépanier est professeur d'histoire à l'Université de Moncton et L. Trépanier est titulaire d'une maîtrise en histoire. Une partie de la documentation à la base du présent travail a été recueillie grâce à une subvention du Conseil de recherches en sciences humaines du Canada. Les auteurs tiennent à remercier $\mathrm{MM}$. H. Decencière-Ferrandière, de Paris, et $\mathrm{F}$. Millou, de Tours, qui les ont beaucoup aidés à comprendre la vie et l'oeuvre de leur grandpère.

1 Louangeurs: A.H. Clark, Acadia. The geography of early Nova Scotia to 1760 (Madison, 1968); R. Roy, La Croissance démographique en Acadie de 1671 à 1763, mémoire de M.A. en démographie (Université de Montréal, 1975). Critiques: M. Brunet, "Trois dominantes...", La Présence anglaise et les Canadiens (Montréal, 1968); M. Roy, L'Acadie perdue (Montréal, 1978).

RHAF, vol. 33, no 3, décembre 1979 
rapports réciproques. Laissant de côté, dans le présent article, les premières, nous nous concentrerons sur la seconde.

Voici dans quel esprit nous abordons notre sujet.

\section{L'HISTORIOGRAPHIE COMME SPÉCIALITÉ}

C'est après bien d'autres que nous nous avançons à l'orée de ce domaine, qui est constitué désormais en spécialité à l'intérieur de la discipline $^{2}$. Quelques notes rapides placeront notre enquête en contexte.

Le passé, qu'on appelle aussi histoire, livre une partie de ses secrets à l'historien qui le sollicite. L'oeuvre qui en résulte - le dialogue entre le passé et le présent - s'appelle aussi histoire. L'ambivalence du terme traduit le rêve têtu des positivistes: faire de l'histoire le reflet en tout point fidèle du passé, comme si, se suffisant à elle-même, elle s'écrivait pour ainsi dire toute seule et réduisait l'historien à la fonction d'intermédiaire, de truchement, de simple révélateur ${ }^{3}$. Le meilleur historien est celui qui disparaît derrière son objet. Or le document historique en soi n'existe pas; il ne s'anime et parle qu'avec l'intervention de l'historien. Alors même que le positivisme était à son zénith, il s'en est trouvé pour s'en aviser. Renan observait: "Il n'y a guère de détails certains en histoire; les détails cependant ont toujours quelque signification. Le talent de l'historien consiste à faire un ensemble vrai avec des traits qui ne le sont qu'à demi." 4 Il entre donc dans l'oeuvre historique une indispensable part de création et beaucoup de subjectivité. L'ensemble vrai qu'avec Renan nous souhaitons ne l'est jamais absolument.

La vérité historique est relative et provisoire ${ }^{5}$. Cela se conçoit aisément. Entre l'histoire-passé et l'histoire-connaissance s'immiscent l'historien et son temps, son métier et son oeuvre. C'est l'historiographie, dont il faut aussi faire l'histoire. Il importe de découvrir comment s'élabore la connaissance historique car, en dernière analyse, l'évaluation de notre compréhension du passé en dépend. Critiquer la science, c'est prévenir l'enfermement dans la

2 C.-O. Carbonell, Histoire et historiens, Une mutation idéologique des historiens français, 1865-1885 (Toulouse, 1976); C. Berger, The writing of Canadian history, Aspects of English-Canadian historical writing:1900-1970(Toronto, 1976); S. Gagnon, Le Québec et ses historiens de 1840 à 1920 (Québec, 1978).

3 Cf. J.-M. Paquette, "François-Xavier de Charlevoix ou la métaphore historienne, Contribution à une systématique du récit historiographique", Recherches sociographiques, 15, 1 (janv.-avr. 1974): 9-19.

4 Vie de Jésus, "Préface".

5 Qu'est-ce qu'un ouvrage définitif? 
science, dont la mission est la libération de l'homme.

Le spécialiste de l'historiographie fait porter son enquête sur deux dimensions subordonnées l'une à l'autre. Il analyse les cadres sociaux de la connaissance historique: les relations entre société, savoir et pouvoir, les rapports entre histoire et idéologie. Il fouille le passé de la discipline historique pour en reconstituer l'évolution méthodologique, la lente accession, par paliers et retombements, à la dignité précaire de science humaine. Il démêle enfin l'écheveau des connexions qui unissent l'une et l'autre dimension. Tâche ardue, peut-être impossible, car il sait que l'idéologie contamine aussi sa propre démarche, que l'anachronisme l'épie et que sa curiosité l'entraîne dans des phénomènes abscons.

Comme la présente étude s'inscrit dans un projet de recherche plus vaste 6 , nous nous contenterons ici d'aborder l'examen d'un aspect de la seconde dimension à savoir comment Rameau percevait le métier d'historien et où il se situait par rapport aux différentes orientations de la discipline à son époque.

\section{DE LA RÉVOLUTION À L'HISTOIRE}

"J'ai été attaché moi aussi aux idéees révolutionnaires", rappelait Rameau en 1877, et, aurait-il pu ajouter, révolutionnaire malheureux, vaincu, je me suis fait historien. Partisan de la révolution de 1848 comme l'était une minorité de catholiques de gauche, adeptes de ce qu'on a désigné d'utopisme petit-bourgeois 8 , Rameau, qui croyait assister à l'aube d'une ère nouvelle ${ }^{9}$, fondée tout ensemble sur la démocratie, la réforme sociale et la restauration de l'esprit religieux dans le peuple, dut se résigner à voir LouisNapoléon relever les aigles de son oncle. L'avortement de son rêve le détermina à quitter l'action. Il lui resta pour la politique un dégoût certain et, pour les hommes qui la font, une méfiance viscérale. En

6 Cf. notre article "À la recherche d'un homme et d'une société: Rameau de SaintPère et l'Acadie", Bulletin du Centre de recherche en civilisation canadienne-française de l'Université d'Ottawa, 16 (avril 1978): 15-17.

7 Archives privées Decencière-Ferrandière (infra ADF), Rameau à F. Schrader, 20 déc. 1877 (brouillon).

8 F. Armand, "1848-1851: grandeur et servitude de l'utopisme petit-bougeois", $L a$ Pensée, nouvelle série, 18 (mai-juin 1948): 48-54.

9 Rameau fut rédacteur à l'Ére nouvelle (ADF, H. Maret, 28 fév. 1849 [lettre d'introduction donnée à Rameau]), puis administrateur-gérant de la République universelle. Cf. J.-B. Duroselle, Les débuts du catholicisme social en France (1822-1870)(Paris, 1951); C. Bressolette, "Civilisation et démocratie chrétienne dans la théologie de l'abbé Maret en 184849", J.-R. Derré et al., sous la direction de, Civilisation chrétienne, Approche historique d'une idéologie (Paris, 1975): 281-299. 
juillet 1853, il reçut l'autorisation de consulter les Archives de la Marine ${ }^{10}$. Il vouerait désormais son existence au travail intellectuel, dans lequel l'histoire occuperait un rang éminent. Propriétaire foncier, rentier à l'aise, ni les loisirs, ni les ressources n'allaient lui faire défaut.

La crise de 1848-51 l'ayant orienté vers l'histoire, ce furent des préoccupations chez lui constantes, depuis le temps de ses études de droit jusqu'à sa mort, qui lui dictèrent ses sujets, ses problématiques et ses méthodes: préoccupations sociales, préoccupations coloniales. Ainsi l'histoire des colonies retiendrait son attention, et il la traiterait sous un angle résolument social. Ses recherches en vue de la publication de sa France aux colonies (1859) excitèrent son intérêt pour l'histoire de la seigneurie ${ }^{11}$. L'évolution de la propriété rurale en France partagea donc ses faveurs avec l'histoire de la colonisation en Amérique ${ }^{12}$. Son grand ouvrage sur l'Acadie (1877, 1889) s'intitulera, logiquement, Une colonie féodale.

Rameau fut un historien autodidacte, comme tous les historiens canadiens-français du XIXe siècle, mais contrairement à ses collègues de France, où l'histoire se professionnalisait depuis la fondation de l'École des Chartes en $1821^{13}$. Il se donna donc à luimême sa formation historique dans les années 1850 et 1860 . Quels maîtres a-t-il pratiqués? Comment, au XIXe siècle, se dessinaient les contours de l'historiographie française?

\section{LA FRANCE HISTORIENNE}

L'histoire s'est prodigieusement développée en France à partir de 1815-1820. Après tant de bouleversements, il fallait raffermir les antiques certitudes historiques, ou les remplacer. La "nouvelle histoire"14, sous la triple pression de l'érudition, du romantisme et des débats idéologiques de la Restauration ${ }^{15}$, a dirigé une part

10 ANQ, Fonds Rameau (infra FR), Rameau au directeur de la Comptabilité générale du Ministère de la marine, 9 mai 1858 (brouillon); Université de Moncton, Centre d'études acadiennes (infra CEA), 2.1.1, Lettre d'autorisation du Ministère de la marine, 7 juin 1859.

11 E. Rameau, La France aux colonies, Études sur le développement de la race française hors de l'Europe, Les Français en Amérique, Acadiens et Canadiens (Paris, 1859).

12 Infra, par. a) La nouvelle histoire et ses maitres.

13 C.-O. Carbonell, op. cit., 251 ss.

14 L'école historique nouvelle, selon Chateaubriand (1831): A. Thierry, “Considérations sur l'histoire de France", Récits des temps mérovingiens... nouvelle édition revue (Paris, [s.d.]), 1: 182.

is A. Thierry, “Considérations...”, 139-40 et passim. 
notable de ses efforts vers le moyen âge. Elle prenait ses distances par rapport aux historiens "systématiques"16 et entendait se consacrer à l'histoire scientifique, c'est-à-dire à la "science qui a pour objet les faits réels et les témoignages positifs"17, "la science de nos origines, des vieilles institutions et des vieilles moeurs" 18 , dont l'essor s'appuyait sur les retrouvailles de l'érudition et de l'historiographie ${ }^{19}$. Elle n'était pas une école, mais un mouvement, divers de tendances - narrative, philosophique - quoiqu'unifié par un but commun, celui de "s'attaquer aux problèmes fondamentaux" 20 .

La nouvelle histoire était surtout libérale; celle du dernier tiers $\mathrm{du}$ XIXe siècle se divisera en deux camps: d'une part, l'école catholique, ultramontaine et légitimiste, avec sa Revue des questions historiques (1866), et, d'autre part, l'école critique, dominée par des historiens protestants, au républicanisme modéré, et groupée autour de la Revue historique (1876) ${ }^{21}$.

Rameau se mit à l'école de la nouvelle histoire en même temps qu'il amorçait son évolution idéologique. Survint alors, dans son existence, une influence essentielle, qui le confirma une fois pour toutes dans son orientation idéologique et dans une certaine pratique du métier d'historien.

\section{L'ÉCOLE DE LA SCIENCE SOCIALE DE LE PLAY}

Rameau avait fait des études juridiques, sanctionnées par un diplôme universitaire en 1842. À son expérience de propriétaire terrien, il avait ainsi ajouté des connaissances techniques susceptibles de servir à un praticien de l'histoire sociale: droit de la propriété, droit de la famille, droit successoral, familiarisation avec une source indispensable: les actes notariés. Lecteur attentif et admirateur des oeuvres de Proudhon ${ }^{22}$, - preuve additionnelle de

\footnotetext{
$16 \quad$ Ibid., 162.

17 Ibid., 200.

18 Ibid., 182.

19 Ainsi on multiplia les publications de répertoires de sources, telle la colossale Collection de documents inédits sur l'histoire de France.

20 A. Thierry, “Considérations...", 182. Thierry admirait Guizot (ibid., 189) et Guizot faisait aussi de la narration. Thierry ne condamnait que les excès de la méthode philosophique (ibid., 200-201). E. Demolins a tenté de concilier les deux tendances dans son Histoire de France (Paris, 1879).

21 C.-O. Carbonell, op. cit., 325ss.

22 F. Millou, Un catholique proudhonien (inédit, vers 1938). Travail intéressant, mais nous avons des réserves.
} 
son intérêt pour la question sociale, de l'ampleur de son information et de la diversité de ses inspirations idéologiques - Rameau fut naturellement amené à s'intéresser aux sciences sociales naissantes. En 1859, il adhéra à la Société d'anthropologie de Paris. C'est muni d'un questionnaire élaboré par cette société qu'il fit son voyage d'Amérique en 1860-61, ses pérégrinations étant toujours studieuses. Au retour, ses observations lui fournirent la matière de communications devant cette Société23. Toujours au début des années 1860 , il collabora à l'Économiste français, publication promotrice de la vocation coloniale de la France et spécialisée dans les questions socio-économiques ${ }^{24}$. Mais tout cela - histoire, anthropologie, économie, colonisation - ne répondaient ni à ses aspirations profondes, ni à ses inquiétudes. Il recherchait non seulement une méthode, mais aussi une doctrine. La Seciété d'Économie sociale, fondée par Frédéric Le Play, lui offrit l'une et l'autre ${ }^{25}$. Il en devint membre en 1865. Le Play éclipsa Proudhon.

Le Play avait mis au point une méthode d'enquête et fondé sa sociologie sur de solides et patientes monographies de familles ouvrières et paysannes. Sur ces données de l'observation, il a édifié une construction idéologique et normative, peut-être fragile à nos yeux, mais qui mérita alors l'approbation admirative de conservateurs et de catholiques intéressés à la théorie et aux aspects pratiques de la question sociale. La sociologie de Le Play se voulait utile: elle entendait découvrir, dans l'étude des familles, la clé et les règles du bonheur des peuples. Dans l'école de Le Play, Rameau découvrait avec enthousiasme le moyen de renouer avec ses préoccupations de jeunesse et de refaire l'unité de sa vie, dans le respect de sa propre évolution qui l'avait rejeté à droite (tendance qu'accentua la Commune ${ }^{26}$. Pour lui, en outre, le mouvement leplaysien venait consacrer la valeur de l'histoire démographique ${ }^{27}$ (qui est en somme celle de la multiplication des familles), de l'histoire sociale (qui

\footnotetext{
Bulletin de la Société d'anthropologie de Paris, 2 (1862): 7ss et 615ss.

J. Duval, "Programme", L'Économiste français, 1, 1 (1861): 1-2.

M.Z. Brooke, Le Play: engineer and social scientist (Londres, 1970). A. Michel, "Les Cadres sociaux de la doctrine morale de Frédéric Le Play", Cahiers internationaux de sociologie, 34 (janv.-juin 1963): 47-68.

26 CEA, 2.1-12, Rameau à E. Dumez, 15 avril 1872 (brouillon).

27 Une partie du succès de Rameau au Canada français s'explique précisément par son insistance sur les faits de population. Québécois et Acadiens percevaient surtout les défis de leur temps en termes de rapports de forces démographiques dans la Confédération et en Amérique du Nord. D'où l'obsession des recensements.
} 
analyse diachroniquement les moeurs des familles), de l'histoire de la propriété foncière (qui étudie les relations entre les familles et la terre patrimoniale où elles s'enracinent ou dont elles s'éloignent pour provigner) et enfin de l'histoire coloniale (qui est une investigation démographique, sociale et foncière sur les familles qui émigrent).

La Société d'Économie sociale faisait large place, dans ses débats et ses publications, à l'histoire et, dans ses rangs, aux historiens. Elle manifestait, pour le grand érudit Léopold Delisle, une véritable vénération et reconnaissait, dans l'École des Chartes, une de ses inspirations fondamentales ${ }^{28}$. Côtoyant, au sein de la Société d'économie sociale, des historiens de tout calibre, pratiquant les érudits qu'elle admirait, Rameau eut l'occasion de parfaire la formation qu'il s'était donnée.

\section{PROPOS DE RAMEAU SUR L'HISTOIRE ET LA MÉTHODE}

Sans jamais avoir écrit de traité, Rameau s'est prononcé sur l'histoire et la méthode dans des notes qui parsèment sa correspondance, ses brouillons et ses publications. Mises bout à bout, elles permettent de reconstituer à peu près fidèlement sa pensée làdessus.

\section{a) La nouvelle histoire et ses maîtres}

Enfin devenue science, se réjouit Rameau, l'histoire, armée de toutes les ressources de l'érudition, aborde les vraies questions historiques:

La science arrive comme les Prières de la Fable, tardive, boîteuse, mais patiente, forte et juste. Ainsi a-t-on refait la Chronique de la France, en reconstruisant d'après les documents originaux, les vraies et secrètes origines de notre force et de nos faiblesses, de nos revers et de nos succès. ${ }^{29}$

Le rôle de l'histoire est "également éloigné des nomenclatures un peu sèches de l'Antiquité, et des déclamations, systèmes déclamatoires de l'histoire politique" 30 . Rameau conteste une certaine pratique du métier d'historien:

28 Voir S. Luce, "F. Le Play, la vieille France, l'École des Chartes et la Société d'économie sociale", La Réforme sociale, 3e série, 2 (1891): 33.

29 FR, Compte rendu de Rameau du livre de Margry sur La Salle.

30 CEA, 2.11-8 (brouillon du compte rendu mentionné à la note 29). 
La Vieille Histoire, solennelle, académique, philosophique et souvent un peu déclamatoire, a certainement son utilité et son mérite, mais elle est insuffisante; surtout lorsqu'elle vit exclusivement sur l'étude des grands personnages, et des intrigues qui s'agitent autour d'eux $[\ldots]^{31}$

Le point de départ de l'histoire doit être les conditions concrètes d'existence des populations, - et non pas "les théories gonflées auxquelles [elles] n'ont jamais songé" et que "nous voulons toujours [leur] supposer", ce qui fait que "nous comprenons mal le passé"32. Il ajoute ailleurs: "Si je ne craignais d'user d'un terme que l'abus a fini par rendre ridicule, je dirais que j'ai voulu faire de l'histoire réaliste." 33

Les maîtres qui ont imprimé à la discipline historique un si décisif essor et qu'ils ont pratiqués, il les énumère lui-même:

Ce sont des hommes tels que Dureau de la Malle ${ }^{34}$, Thierry, Guizot, Lenormand ${ }^{35}$, Guérard ${ }^{36}$ qui ont inauguré cette transformation féconde - et de nos jours, un nombre toujours croissant de continuateurs se pressent sur leurs traces. Je n'ai pas besoin de citer Mrs Clement ${ }^{37}$, Rousset ${ }^{38}$, Wallon ${ }^{39}$, Léopold Delisle, Léon Gautier ${ }^{40}$ et tant d'autres dignes continuateurs. ${ }^{41}$

1878

31 ASQ, Corr. H.-R. Casgrain (infra CC), v. 9, no 65, Rameau à Casgrain, 27 mai

32 Rameau de Saint-Père, Une colonie féodale en Amérique, L'Acadie (1604-1881) (Paris et Montréal, 1889 [1 re éd. 1877]), 1: 14-15.

33 CEA, 2.1-17, Rameau à E. Dumez, 18 fév. 1878 (brouillon). Pour Rameau, Tocqueville était un continuateur de l'histoire à la mode du $18 \mathrm{e}$ siècle.

34 A.-J.-C.-A. Dureau de la Malle (1777-1857), érudit, auteur de travaux sur le moyen âge et l'antiquité, telle L'Algérie, histoire des guerres des Romains, des Byzantins et des Vandales (1852).

35 F. Lenormand (1837-1883), spécialiste de l'antiquité, auteur, entre autres ouvrages, de La Monnaie dans l'antiquité (1878).

36 B.-E.-C. Guérard (1797-1854), directeur de l'École des Chartes, auteur des Prolégomènes du polyptique d'Irminon (1844), oeuvre fort prisée par Rameau.

37 J.-P. Clement (1809-1870), spécialiste de Colbert.

38 C.-F.-M. Rousset (1821-1892), historien catholique dont l'oeuvre porte sur l'histoire militaire de la France.

39 H. Wallon (1812-1904), titulaire de la chaire d'histoire moderne à la Sorbonne, mais aussi auteur de travaux sur Jeanne d'Arc et Saint Louis.

40 L. Gautier (1832-1897), chartiste, animateur avec M. Sepet de la Revue des Questions historiques (catholique et royaliste).

41 CEA, 2.11-8 (déjà cité). 
Augustin Thierry, qu'il admire, a exercé une influence certaine sur Rameau $^{42}$. Fustel de Coulanges l'impressionne aussi ${ }^{43}$. Rameau loue "l'oeuvre magistrale" de Léopold Delisle, "dont l'autorité est si considérable" 44 . Dans sa Colonie féodale, il cite longuement l'érudit et invoque son histoire des classes agricoles en Normandie pour soutenir sa démonstration que les colonies anglaises, françaises et hollandaises de l'Amérique du Nord étaient des prolongements féodaux de leur société d'origine et qu'il ne pouvait en être autrement ${ }^{45}$. Cette thèse, il la résumera d'une phrase: "La seigneurie foncière créait la seigneurie coloniale, comme une ruche émet un essaim." 46

La part des médiévistes dans l'énumération de Rameau ne surprend pas quand on sait que l'histoire coloniale d'Amérique a aiguillé sa curiosité vers l'histoire agraire de France:

L'histoire de la colonisation canadienne et le spectacle que j'avais journellement sous les yeux, durant mon séjour dans le pays, m'avaient singulièrement frappé. Mais ce n'est qu'après mon retour en Europe que j’appris d'une manière claire, l'étroite relation qui existait entre les chartes du moyen âge et la colonisation seigneuriale du Canada, par l'intermédiaire de la coutume de Paris, qui a présidé à l'installation de la Nouvelle-France.

42 “Augustin Thierry a donné le signal de cette réforme historique parmi nous"(CC, v. 9, no 65, Rameau à Casgrain, 27 mai 1878). L'espace nous manque pour énumérer toutes les observations de Rameau qui ne sont que des démarquages des "Considérations sur l'histoire de France", de L'Histoire de la conquête de l'Angleterre, des Récits des temps mérovingiens, des Lettres sur l'histoire de France, etc. - Parmi les bêtes noires de Rameau: Macaulay et surtout H. Martin, qui, bien que disciple de Thierry, n'est qu'un “vieux canard"(CEA, 2: 1-17, Rameau à E. Dumez, 18 fév. 1878 [brouillon]). Le Larousse du XXe siècle trouve ce dernier historien "faible au point de vue critique et scientifique", tandis que le Dictionnaire des dictionnaires de Paul Guérin déplore, chez lui, "le style lourd et l'esprit antichrétien".

43 Auteur de La Cité antique et de L'Histoire des institutions de l'Ancienne France. Rameau a regretté sa "perte prématurée" (Mémoire sur l'origine des grandes propriétés foncières en France (Extrait du Bulletin du Comité des travaux historiques et scientifiques) (Paris, 1893), 2).

44 Rameau, "La Population de la France au moyen âge", Revue des Questions historiques, 23 (1878): 603, 605. L.-V. Delisle (1826-1910), chartiste, archiviste, érudit de très grande classe, ses Études sur la condition de la classe agricole et l'état de l'agriculture en Normandie, au moyen âge ont paru en 1851. "Travail remarquable", selon E. Frère, Manuel du bibliographe normand... (New York, [s.d.]; réimpression de la lre éd., Rouen, 1858-60), 334-335.

45 Rameau, Une colonie féodale, 1: xix, 1-3, 15. janv. 1879.

CC, v. 9, no 62, Rameau à Casgrain, 2 mai 1878; aussi no 86, le même au même, 30 
Je m'appliquai donc, dès l'année 1862, à étudier dans nos vieilles chroniques les phénomènes, économiques et sociaux, analogues à ceux que j'avais observés sur le vif en Amérique. C'est en suivant avec un soin minutieux ces études comparées, que je dois d'avoir appris l'histoire de nos origines rurales et sociales, plus sérieusement que je ne les avais connues sur le banc des écoles. ${ }^{47}$

À partir de cette époque, Rameau mène donc de front ses recherches en histoire acadienne et ses recherches en histoire française. Il projette un grand ouvrage sur l'histoire agraire de la France au moyen âge. Resté en chantier, son travail lui fournira tout de même la matière de quelques communications dans des congrès de sociétés savantes. Il veut démontrer que "le fait primitif et majeur, autrefois en France, a été la petite propriété", c'est-à-dire "la propriété de la terre aux mains de celui qui la travaille" 48 et que, par conséquent, "le Prolétariat dans l'état social est un fait nouveau et non pas le fait ancien de la société"49. Les grands domaines fonciers se sont constitués du XIVe au XVIIIe siècle, par achat continu de parcelles trop subdivisées, ne pouvant plus faire vivre les familles qui les cultivaient ${ }^{50}$. Il s'agit en somme, selon Rameau, pour "les historiens de l'affranchissement du sol et de la division de la propriété au moyen âge [...] [d']achever sur cette époque, l'oeuvre de réparation, et [de] porter le dernier coup à nos préjugés et à notre présomptueuse ignorance" 51 . Cette réhabilitation de l'âge féodal accuse l'itinéraire parcouru par le révolutionnaire de 1848. D'ailleurs, à partir des années 1870 , Rameau a la nette impression que la science est passée du camp libéral au camp conservateur et catholique:

47 Rameau, Mémoire sur les chartes censives au XIe siècle dans quatre Châtellenies (Oise, Cher, Aube et Orne) (Extrait du Bulletin des sciences économiques et sociales du Comité des travaux historiques et scientifiques) (Paris, 1895), 8-9. Rameau reconnaît le rôle de l'intuition (qu'il appelle "prévision instinctive") en histoire: voir CC, v. 11, no 35, Rameau à Casgrain, [sept. 1885].

48 Rameau, "Histoire d'une famille de petits propriétaires du Gâtinais depuis le commencement du XVIe siècle jusqu'à nos jours", Bulletin de la Société internationale des études pratiques d'économie sociale (1875): 721.

49 CC, v. II, no 35, Rameau à Casgrain, [sept. 1885].

so Loc. cit.

51 Rameau, [Intervention lors d'une séance de la Société d'économie sociale], Bulletin de la Société internationale des études pratiques d'économie sociale (1876), 187. 
[...] la jeunesse et les écrivains catholiques y [dans les études historiques] occupent une situation tellement dominante que l'élément purement libre penseur s'en éclipse peu à peu [...] - la plupart des hommes notables sont plus ou moins attachés à cet ordre d'idées - tels que Léopold Delisle, Boutaric $^{52}$, Léon Gautier, Camille Rousset, etc. etc. - Ceux que l'on peut dire indépendants tels que Fustel de Coulanges, Louandre $^{53}$ et autres sont obligés de fausser compagnie à l'École Révolutionnaire et de se tenir à l'écart pour n'être pas compromis par l'ignorance relative dans laquelle elle s'est laissé acculer. ${ }^{54}$

Rassurante évolution, qui double et entérine en quelque sorte le cheminement personnel de Rameau; tableau peu nuancé, mais qui n'est pas absolument sans rapport avec la réalité55.

\section{b) Manifeste pour l'histoire sociale}

Lors de la parution de la première édition d'Une colonie féodale, Rameau commente et explique son ouvrage, sa manière, dans des lettres fort intéressantes adressées à ses correspondants québécois.

Par cet ouvrage, Rameau veut "sortir l'histoire de la colonisation américaine de l'ornière ancienne" où elle s'est enlisée:

[...] on a trop longtemps écrit cette histoire, déplore-t-il, en parlant d'abstractions pures et préconçues, en insistant outre mesure sur les questions purement politiques et militaires; il serait utile de partir aujourd'huy de l'étude des faits et des hommes, et de revivifier l'histoire dans ces observations curieuses et fécondes que présente l'étude des populations, des familles elles-mêmes, qui sont la trame essentielle des sociétés que l'on décrit. 56

52 E. Boutaric (1829-1877), professeur à l'École des Chartes, auteur de la France sous Philippe le Bel (1861), membre de l'Académie des inscriptions et belles-lettres.

53 C.-L. Louandre (1812-1882), à La Revue des Deux Mondes, puis au Journal général de l'instruction publique, auteur d'un Essai sur le mouvement communal dans le Ponthieu (1837).

54 CEA, 2.1-17, Rameau à E. Dumez, 18 fév. 1878 (brouillon).

55 C.-O. Carbonell ( $o p$. cit.) met au jour le rôle considérable joué par les catholiques dans le monde des historiens français vers 1865-1885.

56 ANQ, Fonds Siméon Le Sage (infra FSL), Rameau à Le Sage, 25 fév. 1878. 
Cette insistance sur la famille, comme catégorie sociale de base, révèle sans doute l'influence de Le Play, mais elle correspond aussi très exactement à la conception de l'histoire qu'a Rameau: on ne saurait se satisfaire d'une superficielle chronique politico-militaire, occupée à démêler les "intrigues de quelques grands personnages" ou à soupeser les "commérages politiques du temps passé" 57 . Le mérite de cette "histoire toute nouvelle" - l'étude diachronique des familles - n'est pas uniquement d'être "plus vivante et plus pittoresque" 58 . En effet, elle seule permet de saisir

complètement la physionomie réelle, et les mystères de l'histoire du passé. On connaît alors le secret véritable de la force et de la faiblesse des nations, parce que l'on pénètre dans leurs moeurs, dans leurs idées, dans leurs croyances, dont l'influence est bien autrement forte sur leur destinée que celle de leurs institutions et constitutions. Le 18ème siècle avait semé sur ce sujet comme sur bien d'autres, les plus fausses idées, et plus nous travaillons aujourd'huy, plus nous nous apercevons que toute l'histoire qui a été écrite sous l'influence des préjugés ampoulés de cette école est entièrement à refaire..$^{59}$

Ici on sent bien que l'idéologue vient prêter main forte à l'historien. L'explication est à chercher dans l'itinéraire personnel de Rameau, qui était bien revenu de ses enthousiasmes politiques de 1848$1851^{60}$. Chaque génération d'historiens fournit - le plus souvent avec le renfort de l'idéologie - son contingent de révisionnistes.

Ainsi la conception qui est la sienne invite Rameau à hausser l'histoire au niveau de l'histoire-problème ${ }^{61}$.

57 Loc. cit.

58 Loc. cit.

59 CC, v. 9, no 65, Rameau à Casgrain, 27 mai 1878.

60 CEA, 2.1-9, Rameau à E. Dumez, juin ou juill. 1868 (brouillon).

61 Voir G. Massicotte, "L'historiographie française de la question ouvrière: réflexion de méthode, La théorie de la connaissance dans quelques ouvrages récents", Histoire sociale, 11, 21 (mai 1978): 183-214. 
La problématique retenue par Rameau est triple:

10 exposer comment la colonisation américaine se rattachait à l'état social de la Vieille Europe;

$2^{0}$ offrir un spécimen en action des procédés et des formes qui caractérisèrent cette colonisation;

$3^{0}$ montrer quelle était la valeur réelle de nos colons méconnus et des colonies que nous avons si malheureusement et si maladroitement perdues. ${ }^{62}$

Cette problématique - on le constate — s'inspire de soucis multiples: considérations proprement scientifiques, préoccupations idéologiques et visées utilitaires. La présence de ces trois dimensions ne nous paraît pas imputable à une idiosyncrasie particulière, mais bien inhérente à la condition historienne même.

Enfin, Rameau se déclare partisan de la méthode comparative "car l'histoire ne se connaît bien que par relation et comparaison"63. D'où ses rapprochements entre les expériences coloniales françaises, britanniques, espagnoles et hollandaises; ses parallèles entre la seigneurie en France et la seigneurie en Angleterre, et leur contrepartie respective dans les colonies ${ }^{64}$.

\section{c) Les sources de l'histoire sociale}

Autre histoire, autres sources. Les documents officiels, les dépêches ministérielles ou diplomatiques ne suffisent plus. Il faut puiser dans les papiers de familles (vieux titres, vieux registres, vieilles correspondances), les traditions orales ${ }^{65}$, les légendes mêmes 6 :

Ces sortes de documents forment un complément très fécond aux documents officiels - en donnant à ceux-ci une physionomie de l'époque. Ces derniers fournissent une forme, un programme, auxquels les premiers apportent en quelque

62 CC, v. 9, no 55, Rameau à Casgrain, 22 janv. 1878. FSL, Rameau à Le Sage, 25 fév. 1878: "J'ai pu jeter sur tout cela quelques artifices de style ou de récit, pour rendre l'ouvrage plus lisible, mais mon but sérieux et réel le voilà."

${ }_{63}$ CC, v. 9, no 86, Rameau à Casgrain, 30 janv. 1879.

64 CEA, 2.2-13, brouillon d'une étude de Rameau sur le morcellement comparé des terres en Angleterre et en France au Moyen âge. Il est aussi l'auteur de "L'Expansion des Franco-Canadiens et la colonisation française en Algérie, Etudes comparées d'histoire contemporaine", La Réforme sociale, 2e série, 8 (1889): 654-667.

65 FSL, Rameau à Le Sage, 6 mai 1887.

$66 \mathrm{CC}$, v. 1, no 91, Rameau à Casgrain, 20 oct. 1861. 
sorte la vie en reproduisant l'aspect et le mouvement de l'époque. Nous les considérons aujourd'huy dans les recherches historiques comme un des éléments les plus précieux de l'histoire $[\ldots]^{67}$

On ne doit pas non plus négliger les recensements nominaux et les généalogies, les minutes d'actes civils ou religieux conservées dans les archives notariales, municipales et paroissiales ${ }^{68}$. Dans sa correspondance, Rameau se présente comme un historien consciencieux, qui met tout en oeuvre pour réunir la documentation la plus complète et la plus pertinente possible. Si ses études l'avaient initié à la langue du droit, en revanche il n'avait pas reçu l'entraînement des charistes. C'est en autodidacte qu'il a dû apprendre à déchiffrer les vieux actes notariés "écrits cursivement et très serrés", à se familiariser avec les "abréviations qu'on y emploie"69. Comme le prouvent ses voyages en Amérique, en 1860-61 et 1888, il ne s'est pas borné à l'information médiatisée des manuscrits et des études: il voulait avoir prise directe sur son objet. Pour son histoire de l'Acadie (Une colonie féodale) qu'il projetait dès 1859 , il souhaitait "aller voir [lui]-même les lieux, et faire un petit séjour au milieu des divers débris de cette race infortunée pour mieux [s'] informer de leurs traditions et mieux connaître leur situation présente"70. Il s'imposait aussi des déplacements en France pour mener des enquêtes d'histoire orale. Ainsi, s'étant rendu dans le Perche, il y découvrit "une famille Turgeon qui avait toujours conservé la tradition du départ d'un de ses membres, Charles Turgeon, qui en 1663 était parti de Mortagne pour aller rejoindre Mr Giffard à Beauport avec toute sa famille"71. Les recherches de Rameau paraissent donc répondre aux critères les plus exigeants de la méthode historique.

Devant le document, Rameau gardait une certaine liberté. Il n'en faisait pas un absolu'2 . Il avait le sens du relatif et reconnaissait

67 FSL, Rameau à Le Sage, 6 mai 1887.

FSL, Rameau à Le Sage, 25 sept. 1880.

Loc. cit.

ADF, Rameau à E. Parent, 14 fév. 1860 (brouillon).

FSL, Rameau à Le Sage, 25 sept, 1880 .

72 Rameau, "M. Rameau et 'la Nation"', L'Opinion publique, 6 juin 1878: "Il ne suffit pas, en effet, d'adorer les documents, ni même de les collectionner; l'antiquaire monomane possède lui aussi beaucoup de documents [...]; si l'on déplace une syllabe, une motte de terre, il crie qu'on écorche l'histoire. L'historien, cependant, n'est pas si rigoriste, il parle moins de documents, mais il les possède mieux; $[\ldots]$ il les comprend, les contrôle et les complète; [...] le premier est simplement le porteur des reliques, le second les discute, il en sait l'usage et la valeur." 
que l'anachronisme guette toujours l'historien: "N'oublions donc jamais que, pour bien juger les faits et les hommes du passé, au lieu de les amener à nous, il faut aller vers eux, nous mettre en leur place et nous pénétrer de leurs idées et de leurs habitudes." "73 Sans l'historien qui l'interprète, le document ne vaut rien: là est l'exaltant, mais aussi le difficile du métier: découvrir tout ce qui se cache dans le document et rien qui n'y soit pas. Par conséquent, ce que recèle le document dépend beaucoup de celui qui l'interroge. Aussi, alors que l'encre d'Une colonie féodale n'était pas encore sèche, Rameau rêvait-il à une seconde édition où il pourrait, "tout en complétant le récit historique, y joindre 100 pages de documents qui donneraient à [s]on travail une grande assiette" 74 . Comme Thierry, il affectionnait les pièces justificatives à la fin des livres d'histoire: “C'est, en effet, la présence des actes originaux qui constitue, pour une grande part, l'autorité et la fécondité des oeuvres historiques." 75 Il s'en expliquait ainsi. Le lecteur critique veut qu'on lui mette sous les yeux "la sincérité du document tout nu" parce que, premièrement, "les faits que nous racontons portent toujours plus ou moins quelques reflets de nos idées, de nos sentiments, et surtout du point de vue auquel nous nous sommes placés en lisant les manuscrits" et que, deuxièmement, "ce serait [...] une grande erreur de croire que le même document dise la même chose pour tout le monde"76. Par conséquent, la reproduction de documents ne peut que servir l'histoire "puisqu'ils facilitent [...] cette variété d'aperçus qui fécondent la science et contrôlent ses assertions"77. Remarquable lucidité.

Le souci de la vérité historique poussait Rameau à solliciter critiques et corrections ${ }^{78}$. À l'auteur d'un compte rendu d'Une colonie féodale, qui avait laissé entendre que Rameau avait peutêtre sélectionné ses documents, ce dernier écrivit:

J'ai toujours été porté au contraire à étudier de préférence les travaux de ceux qui ne pensent pas comme moi, c'est le meilleur et le plus sûr aiguillon du travail intellectuel. [...] Il eut donc été contraire à la pratique de toute ma vie de cribler des

73 Rameau, Une colonie féodale, 1; 12.

FSL, Rameau à Le Sage, 25 fév. 1878.

Rameau, Une colonie féodale, 1: vii.

Ibid., 1: vii-viii.

Loc. cit. De concert avec l'abbé Casgrain, Rameau a publié les "Registres des Acadiens de Belle-Isle-en-mer" dans Collection de documents inédits sur le Canada et l'Amérique publiés par le Canada-Français (Québec, 1888-1890), 3 v.

${ }_{78}$ ADF, Rameau à E. Parent, 14 fév. 1860 (brouillon). 
documents, et la critique souvent sévère que j'ai faite du gouvernement de Louis 14 et autres, vous montrera assez que je peux dire la vérité à tous.

Si donc il était à votre connaissance que j’aie laissé de côté des autorités considérables dans l'exposition des faits, je vous serais bien reconnaissant de m'indiquer les points précis où je me serais trouvé en défaut, même les simples suspicions qui auraient pu s'élever dans votre esprit [...], et dans le cas où je serais appelé à publier une seconde édition de ce travail, je regarderais comme mon devoir - ou de les discuter, ou de me rectifier. C'est un des principes de l'école positiviste à laquelle j'ai appartenu, et à laquelle j'appartiens encore dans une certaine mesure. ${ }^{79}$

La rigueur du travail historique exige que l'historien, au besoin, se fasse violence, bride sa sensibilité. Rameau avouait qu'il avait dû se refréner, au cours de la rédaction de la France aux colonies, dont le but était d'étudier le "développement progressif de la population et des faits qui s'y rattachent": il fallait résister à la tentation de s'écarter de ce programme en dépit de la fascination qu'exerçaient sur lui "les drames pittoresques, bizarres, émouvants" 80 qui abondent dans l'histoire québécoise et surtout acadienne.

\section{d) Un champ en friche: l'histoire acadienne}

La rédaction de la France aux colonies révéla à Rameau l'histoire acadienne. Mais la part des chiffres, le but qu'il s'était assigné dans ce livre lui interdisaient de narrer par le menu l'histoire des Québécois et des Acadiens. Pour ceux-là, il n'y avait pas grand inconvénient puisque leur passé était reconstitué dans des histoires "dont celle de Mr Garnaut [sic] est même fort remarquable"; pour ceux-ci toutefois, "dont l'histoire est inconnue", cela tirait plus à conséquence. Aussi, quand parut Une colonie féodale (1877), il y avait déjà dix-sept ans que Rameau en caressait le projet. Dès 1860 , il confiait à Étienne Parent, en parlant des Acadiens:

79 ADF, Rameau à F. Schrader, 20 déc. 1877 (brouillon).

80 Rameau, La France aux colonies, xiii. Dans Une colonie féodale, il paraît avoir eu moins de succès dans ses efforts pour tenir la bride à ses sentiments. 
[...] j'ai tellement conscience d'avoir été au-dessous de mon sujet que je ne pourrais me pardonner à moi-même d'avoir éclopé ainsi leur histoire, si je ne faisais voeu de recommencer entièrement l'histoire [...] navrante de ce peuple industrieux et héroïque pour lequel la France a été une marâtre et l'Angleterre un bourreau. ${ }^{81}$

Attrait d'un sujet de choix, aussi désir de combler de graves lacunes dans l'histoire des établissements en Amérique de la France d'autrefois, en vue du progrès des connaissances sans doute, mais encore afin d'éclairer et de presser la marche de l'expansion coloniale de la France de son temps - science et idéologie se renforçant l'une l'autre, comme il arrive souvent. Rameau déplorait "le peu de connaissances que nous avons des affaires de l'Amérique, et en particulier de son histoire qui est très peu familière même à nos savants"82. Selon lui, à part L. Dussieux, "un des hommes rares en France qui s'intéress[ai] ent au Canada"83 et C. Moreau ${ }^{84}$, il n'y avait guère que $P$. Margry à étudier sérieusement l'histoire de la Nouvelle-France. Il tenait cet érudit en haute estime:

Mr Margry relève de cette grande école [des Thierry, Guizot, Delisle et autres], il a senti le besoin de faire pour les études coloniales, ce que tant d'illustres écrivains avaient réalisé pour l'histoire de France. Trente années de travail assidu au milieu de nos archives, une correspondance étendue avec toutes les parties du monde, lui ont assuré une érudition consommée à laquelle recourent aujourd'huy tous ceux qui étudient sérieusement les annales de nos colonies. ${ }^{85}$

Parmi les historiens québécois, on peut dire que Rameau a eu deux maîtres: F.-X. Garneau et, surtout, J.-B.-A. Ferland. À preuve, cette confidence en 1878 :

81 ADF, Rameau à E. Parent, 14 fév. 1860 (brouillon).

82 FSL, Rameau à Le Sage, 25 fév. 1878. Voir aussi FR, Rameau à B. Sulte, 12 ou 13 janv. 1878; ASQ, Fonds Verreau 33, no 91, Rameau à P.-J.-O. Chauveau, 3 fév. 1878.

83 Fonds Verreau 33, no 90, Rameau à H.-A. Verreau, 6 fév. 1874. Dussieux est l'auteur du Canada sous la domination française d'après les archives de la Marine et de la Guerre, 3e éd. (Paris, 1883; 1 re éd., 1855). (Paris, 1873)

${ }^{84}$ Auteur de L'Histoire de l'Acadie française, Amérique septentrionale, 1598-1755

85 FR., Compte rendu de Rameau du livre de Margry sur La Salle. 
C'est l'excellent et savant Abbé Ferland qui je crois a le premier utilisé largement -cette source historique [les registres paroissiaux], en tout cas c'est lui qui m'a appris à m'en servir, et qui m'a fourni beaucoup de données qui en proviennent. ${ }^{86}$

À preuve aussi, cet extrait de l'Économiste français où Rameau présente Ferland comme "un érudit plein d'esprit et de finesse, professant l'histoire comme un bénédictin et spirituel comme un fantaisiste"87. Même si ses préférences allaient à Ferland, il reconnaissait, chez Garneau, "le talent sobre et distingué" qui, ajoutait-il, "n’a guère été dépassé en Amérique que par les beaux travaux de William Prescott"88. Rameau apprit l'existence de Garneau et de son Histoire par la lecture des notes de voyage en Amérique de J.-J. Ampère parues dans la Revue des Deux Mondes du 15 janvier $1853^{89}$. Il écrivit pour la première fois à Garneau en 1858 pour lui demander des renseignements sur la population et l'état socio-économique des établissements francophones d'Amérique. Dans une lettre de ton à la fois déférent et cordial, il faisait appel à "l'intérêt commun qui nous guide pour l'union de toutes les fractions de la race française et la conservation du bon souvenir de nos communes origines"90. Il opposait à l'oeuvre de Garneau, "austère et philosophique, se tenant dans les sphères élevées d'où l'on embrasse les faits généraux", l'histoire de Sulte, qui en était selon lui le complément:

M. Sulte considère les groupes locaux, la famille comme la base élémentaire, la molécule de l'histoire, système original par lequel on étudie les sociétés humaines, comme font les chimistes pour les métaux, en observant la forme des cristaux atomiques qui les composent. C'est ainsi qu'après avoir acquis une connaissance intime de la famille et de la vie sociale, on reconstruit avec science et patience les grandes séries historiques, les grands phénomènes sociaux et les nations qui ne sont que l'agglomération des familles superposées par le cours des âges. 91

86 CC, v. 9., no 65, Rameau à Casgrain, 27 mai 1878. Rameau admirait aussi beaucoup Le Dictionnaire généalogique de l'abbé Tanguay (Rameau, La littérature canadienne (1878-1888), Extrait du Compte rendu des travaux du Congrès bibliographique international (Paris, 1888), 6).

${ }_{87}$ L'Économiste français, 1, 6 (25 fév. 1862): 71.

43) et de The conquest of Peru (1847)

89 Archives privées des auteurs, note manuscrite de Rameau (don de M. F. Millou).

90 FR, Rameau à F.-X. Garneau, 15 juill. 1858 (brouillon).

91 Rameau, La littérature..., op. cit., 5. 
Sulte, francophobe invétéré, faisait exception pour Rameau, avec qui il se lia d'amitié et en qui il voyait le point de départ d'une renaissance de l'histoire coloniale en France, l'un des fondateurs d'une "nouvelle école historique": "On fait la guerre aux vieilles méthodes des écrivains et les recherches se portent vers le fond des choses." 92

Quant aux relations avec B. Murdoch et Th. B. Akins, bien que sporadiques, elles paraissent avoir été empreintes de cordialité. Murdoch mourut avant de recevoir l'exemplaire d'Une colonie féodale dont Rameau lui fit hommage en y joignant "l'expression de ma reconnaissance pour les lumières que vous m'avez fournies, et mes remerciements pour la bienveillante sympathie que vous m'avez montrée"93. Dans cette lettre, il affirmait que l'ouvrage de Murdoch lui avait été d'un grand secours. Les différences entre cet ouvrage et le livre de Rameau ne tenaient pas seulement aux "sentiments de nationalité qui ont pu influer sur chacun" des auteurs, mais aussi au fait que Rameau avait voulu établir le lien étroit entre l'état social préexistant en métropole et celui des colonies naissantes. Dépassant le cas de "la poétique mais petite Acadie", il lui avait été facile de prouver l'influence de la féodalité dans les autres colonies françaises d'Amérique, la Virginie et la Nouvelle-Hollande. Rameau admettait s'être un peu aventuré dans le cas de la Nouvelle-Angleterre, et faisait appel à "l'érudition expérimentée" de l'historien néo-écossais: pourquoi ce dernier n'entreprendrait-il pas des études analogues? appuierait-il sa propre thèse, selon laquelle la société puritaine était moins une démocratie qu'une oligarchie théocratique?

À Akins, nous n'avons trouvé qu'un brouillon de lettre où Rameau regrette de ne pas l'avoir rencontré lors de ses recherches à Halifax. Aucune amertume dans ces lignes, mais des remerciements pour la collection de documents éditée par l'archiviste et l'expression du désir de lui rendre service ${ }^{94}$.

Comme on l'a suggéré plus haut, pour Rameau, l'histoire ne remplissait pas seulement une fonction de connaissance: elle avait une portée pratique.

92 B. Sulte, L'Acadie française, 2e éd., publiée par G. Malchelosse (Montréal, 1930), 16-17. anglaise).

93 FR, Rameau à B. Murdoch, 10 août 1877 (brouillon en français et traduction

CEA, 2.1-20, Rameau à [T.B. Akins, 18 oct. 1882?]. Cf. B.C. Cuthbertson, "Thomas Beamish Akins: British North America's pioneer archivist", Acadiensis, 7, 1 (automne 1977): 86-102. 


\section{e) De l'utilité de l'histoire}

Rameau préconisait une histoire scientifique, rigoureuse, étayée d'une solide preuve documentaire. Mais il n'en découlait pas, selon lui qu'elle ne dût être pour autant qu'une "satisfaction intellectuelle". L'histoire devait se mettre au service des générations présentes et futures: contribuer à la solution de la question sociale, de la question coloniale et de la question nationale. Rameau croyait à la puissance de l'histoire. Qu'elle pût être une "force patriotique", l'histoire de l'Allemagne le montrait assez, dont l'unification a été patiemment mais sûrement préparée par ses historiens ${ }^{95}$. Québécois et Acadiens devaient aussi chercher dans l'histoire un moyen de réaliser leurs destinées:

Montrer tout ce qu'il y avait de forces sociales, intellectuelles et morales en germe dans les temps primitifs du Canada; faire voir comment des circonstances fortuites en ont retardé le développement, et reporté à longue échéance le résultat des promesses que contenait l'aurore; signaler aux Canadiens ce qu'ils peuvent faire pour poursuivre l'essor logique de cette destinée que leur présageaient la sagesse et la vertu de leurs ancêtres, voilà, ce me semble, quel doit être l'objectif de l'histoire du Canada aujourd'hui, si l'on veut que l'étude de l'histoire soit non seulement la fantaisie intellectuelle de quelques antiquaires, mais un enseignement vivant et fécond dans lequel les peuples sages et intelligents vont préparer les forces de leur avenir par l'observation du passé. ${ }^{96}$

Le savoir historique doit être un pouvoir rassembleur "sur le terrain fécond du patriotisme, dans l'étude d'un passé glorieux, commun à toute la Nation, et qui doit servir de base à son avenir"97.

L'histoire n'a rien à voir avec la passion de la nécrophilie; elle débouche sur l'action, sur la vie. "L'histoire est-elle une simple collection de faits, ou une science d'observation qui nous fournit d'utiles enseignements pour notre conduite?"98 Or l'histoire des Canadiens français est particulièrement "féconde en enseignements et en exemples"99. C'est pourquoi Rameau se félicitait tant que ses

\footnotetext{
95 Lettre de Rameau à B. Sulte citée par ce dernier dans L'Acadie française, 63-64. Loc. cit.

CC, v. 9, no 55, Rameau à Casgrain, 22 janv. 1878.

CEA, 2.1-9, Rameau à E. Dumez, juin ou juill. 1868 (brouillon).

CC, v. 1, no 91, Rameau à Casgrain, 20 oct. 1861.
} 
travaux historiques aient attiré sur la Nouvelle-France l'attention de la "vieille France, trop oublieuse de ceux qu'elle n'a perdus après tout que par la négligence, et son futile amour du plaisir"100. Les Français devraient se pénétrer des leçons que leur prodiguent, par leur histoire, leurs "compatriotes du Canada qui moins brillants peut-être dans leur petit nombre et leur délaissement, valent bien mieux qu'[eux] sous beaucoup de rapports" 101 .

Quel est donc "le fonds [sic] essentiel de cette histoire"? "Le développement des Canadiens, et leur progression plus ou moins rapide" en dépit de la défaite, de la conquête et même, dans le cas des Acadiens, de la déportation. Et ce développement, cette progression sont à la fois cause et conséquence de "leur puissante moralité". "Sans moralité, expliquait Rameau, sans ferveur religieuse, on n'aurait pas vu cette multiplication des familles, ni la force que ces nombreux enfants ont donnée à la Nation."102 Là, dans cette "évidente corrélation" - et il y insistait - résidait l'enseignement essentiel de l'histoire canadienne, dont il ne fallait pas varier. Vers la fin de sa vie, Rameau sentait le besoin de multiplier les mises en garde, de plus en plus pressantes ${ }^{103}$. À mesure que les Canadiens français lui semblaient s'éloigner des vertus des "vieux temps hérö̈ques du Canada"104, montait en lui le doute bientôt lancinant qu'ils n'étaient peut-être plus tout à fait, comme il l'avait proclamé dix ans plus tôt, "l'expression la plus virile du génie et [de] la puissance française" 105 .

Ainsi Rameau n'admettait pas que l'historien s'abstînt de faire suivre sa plongée dans le passé d'un retour sur le présent et d'un effort pour éclairer l'avenir. Pour cela, l'historien devait dominer l'histoire, juger de l'orientation des lignes de faîte, donc accéder à la philosophie de l'histoire. Et la philosophie de l'histoire que Rameau s'était donnée était celle d'un moraliste chrétien.

Son système s'appuyait sur le refus du positivisme. Le point de départ reste - doit rester - "l'observation des faits, cette source

\footnotetext{
100 FR, Rameau à P.-J.-O. Chauveau, 11 juin 1859 (brouillon).

Loc. cit.

102 CC, v. 20, no 106, Rameau à Casgrain, 19 avril 1897. D'où l'importance des recensements.

103 Loc. cit. Méfaits de l'américanisation.

104 CC, v. 17, no 108d, Rameau à Casgrain, 23 juin 1894.

105 CC, v. 11, no 195, Rameau à Casgrain, ler juill. 1887.
} 
inépuisable de vigueur intellectuelle comme la Terre qui fortifiait Antée"106! Mais refuser de transcender les faits, ce serait

tomber dans ce grossier positivisme où plus d'une intelligence distinguée va sombrer aujourd'huy; il est des gens qui sont fort disposés aujourd'huy à ne reconnaître de sciences que celles qui peuvent tomber sous le sens le plus vulgaire; ils s'imaginent que pour gouverner le monde il suffit de connaître les lois de la mécanique, et la science des $\mathrm{x}$; mais j’ai souvent observé que tous ces négateurs de rêverie et d'idéal sont proprement si enivrés de leurs particulières rêveries, qu'ils s'écartent du sens commun, généralement adopté par l'humanité, ou bien affolés par leur inaptitude de poser des questions abstraites, ils trouvent qu'il serait commode de noyer leur ignorance propre dans une ignorance générale par la négation même des sciences métaphysiques. ${ }^{107}$

Si Rameau ne pouvait se rallier au positivisme vulgaire, c'était que, pour lui, l'histoire avait un sens et que ce sens prenait sa source en Dieu. Le providentialisme, bien qu'il se fît discret dans ses écrits proprement historiques et n'intervînt guère dans la chaîne causale, n'en était pas moins le fondement même de sa philosophie et, par conséquent, de son interprétation de l'histoire ${ }^{108}$. Voici comment Rameau posait les rapports du providentialisme avec la liberté des agents de l'histoire, individuels ou collectifs:

[...] au-dessus de toutes [les formes politiques] je place la Providence. Quel autre moteur pourrait diriger toute cette masse d'événements, et de ressorts qui agitent et entraînent l'humanité dans son développement? Ce développement nous en apercevons très bien l'enchaînement lorsque l'histoire nous le montre, et nous le percevons assez mal lorsqu'il se passe sous nos yeux; cela ne suffirait-il pas à démontrer la parfaite impuissance de la volonté ou des desseins de l'homme, non pas dans sa conduite privée, non pas dans la direction plus ou moins sage des sociétés, mais dans le gouvernement général de la machine? 109

Tel qu'il le concevait, le providentialisme n'entraînait donc "ni scepticisme, ni résignation abjecte. Nous affirmons que l'homme est

106 CEA, 2.1-9, Rameau à E. Dumez, juin ou juill. 1868 (brouillon).

107 Loc. cit.

108 C'est notre conviction que tout historien a une philosophie de l'histoire et que cette dernière intervient toujours dans la construction historienne.

109 CEA, 2.1-9, Rameau à E. Dumez, juin ou juill. 1868 (brouillon). 
libre dans sa volonté propre, en même temps que nous avouons qu'il est borné dans sa puissance d'action par une puissance supérieure." 110

Bon gré mal gré, les historiens n'échappent jamais tout à fait au monde des valeurs. Les jugements peuvent se feutrer, ils n'en restent pas moins partie intégrante de la construction historienne. Aussi est-il toujours révélateur de connaître la morale - c'est-à-dire la hiérarchie des valeurs - de l'historien. Comme toute échelle de valeurs repose plus ou moins sur l'idée de Progrès (ne serait-ce que pour la réfuter), il importe d'évoquer les convictions de Rameau làdessus.

Rameau récriminait-il contre "les progrès matériels de l'humanité"? "Aucunement, répondait-il; seulement le Progrès pris en général me semble incomplet et mal équilibré; c'est là ce qui cause mon souci." 111 L'expérience de l'histoire enseigne, expliquait-il, que "la dissolution ordinaire des sociétés [a] précisément posé ses prodromes au moment même du maximum de la richesse sociale et des progrès matériels" 112 . Et cela vient d'un défaut d'équilibre entre le progrès matériel et la moralité: plus une société est opulente, plus elle a besoin d'être vertueuse. En un mot:

L'Humanité est forte ou faible selon que les hommes dont elle se compose sont eux-même[s] vigoureux ou amoindris; l'excellence du gouvernement ni des lois ne saurait rien y changer et la meilleure constitution du monde ne ferait point faire bonne figure à un peuple abâtardi. ${ }^{113}$

Elle serait inutile la législation que l'on voudrait imposer aux hommes pour les moraliser: ils doivent "se commander intérieurement et mettre sous le joug d'une direction raisonnable, méthodique, leurs désirs et leurs entraînements"114. "Telle est, concluait-il, l'alliance raisonnée de la liberté et de la répression dans ce que chacune à d'utile."115

$$
\begin{array}{ll}
110 & \text { Loc. } \text { cit. } \\
111 & \text { Loc. cit. } \\
112 & \text { Loc. cit. } \\
113 & \text { Loc. cit. } \\
114 & \text { Loc. cit. }
\end{array}
$$

115 Loc. cit. Pour une autre discussion de l'idée de Progrès, voir CEA, 2.1-12, Rameau à E. Dumez, 15 avril 1872 (brouillon): "Famille - liberté - esprit d'ordre et de travail - modestie et prudence - défiance de son propre sens - lutte contre soi-même basés sur la conscience et la responsabilité individuelle. Voilà les vertus qui ont fondé les grandes sociétés - et que la religion à travers ses abus nous a toujours enseigné[es]." 


\section{CONCLUSION}

Parce que Rameau a beaucoup écrit et qu'une partie précieuse de sa correspondance et de ses notes nous est parvenue, il se livre à nous avec une particulière transparence. Nous n'avons pas à juger sa philosophie de l'histoire, son idéologie, mais à déceler leur influence sur l'histoire qu'il a écrite, ce qui fournira la matière d'un autre article. Nous ne pouvons non plus ici évaluer le degré de fidélité de son oeuvre au programme qu'il vient, en quelque sorte, de nous exposer lui-même: la rigueur qui, à son avis, doit imprégner tout travail historique digne de ce nom; l'accent très moderne qu'il met sur l'histoire sociale. Nous avons montré que cette insistance s'inscrivait dans l'évolution des sciences humaines à son époque. Mais cette explication seule ne satisfait pas: elle appelle un complément qui est à chercher dans l'idéologie.

L'itinéraire idéologique de Rameau a façonné peu à peu son esprit et sa curiosité de telle façon que les phénomènes sociaux occupent à ses yeux la première place. Il avait cru un temps que la politique était le moyen par excellence de reconstruire la société pour y instaurer la liberté et l'égalité, pour y restaurer l'union du peuple et de la religion. Puis il s'était convaincu que les termes devaient être inversés: la politique ne peut structurer la société, dont elle n'est en somme qu'un reflet; c'est au contraire la religion et la famille qui constituent l'ordre politique et c'est sur elles qu'il faut faire porter ses efforts. Si de l'état social et religieux dépend le bonheur des peuples, il importe d'en étudier la genèse. Or - Le Play le lui enseigna - le destin de la famille est lié au patrimoine foncier dont elle vit. Religion, famille et propriété sont les trois structures sociales fondamentales; en analyser méthodiquement l'évolution, dans la diachronie et la synchronie, c'est saisir la société en son centre vital. Le reste n'est que l'écume.

Si Rameau s'est passionné pour l'histoire du Québec et de l'Acadie, ce n'est pas seulement parce qu'il y trouvait "un des types les plus parfaits de la colonisation par immigration" 116 . C'est aussi, c'est surtout parce qu'elles lui apparaissaient - l'Acadie en particulier - comme paradigmatiques. L'Acadie était, pour ainsi dire, la démonstration vivante de la véracité de son système: elle avait survécu non seulement sans pouvoir politique, mais même

116 CC, v. 9, no 60, Rameau à Casgrain, 24 mars 1878. 
contre les puissances politiques, ne devant son "étonnante conservation" qu'à son "énergie et à la bienfaisante influence des vertus chrétiennes"117, dont la fécondité des familles lui semblait le plus beau fleuron. Religion, famille et propriété rurale sauvèrent ce qu'avait compromis l'État. Exaltante preuve que le raccourci politique est un leurre, rassurante confirmation du cheminement intime de l'ancien radical catholique de 48 . Enfin, la France d'Amérique offrirait sa société exemplaire à l'admiration de la France d'Europe, qui y pourrait découvrir le secret de son relèvement.

Le modèle d'histoire sociale mis au point par Rameau n'est pas sans faille. Il escamote une dimension capitale de la vie des sociétés: celle des conflits. Sa pensée repose sur un consensus social à établir par la religion, la tradition et la modération des désirs. Trop exclusivement axée sur la propriété rurale et la famille, elle négligeait la classe, le groupement d'intérêts, ainsi que la société industrielle montante, avec ses villes, ses fortunes mobilières et son salariat.

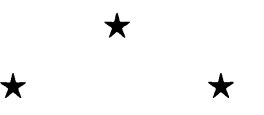

Ni Jean-Paul Hautecoeur, ni Michel Roy n'ont mésestimé l'importance de l'historiographie comme fondement de l'Acadie du discours 118 et de l'Acadie perdue. Mais la tendance révisionniste n'a pas à s'embarrasser de perspectives historiques. Ce n'est pas son propos. Elle a placé Rameau sur la sellette. Une idéologie a fait le procès d'une autre. Notre point de vue diffère. N'ayant pas de compte à régler avec le vieil érudit, nous l'avons longuement interrogé sur sa conception du métier d'historien. Il nous a répondu - avec la sincérité qui convainc - que, tout en rejetant l'illusion de l'objectivité absolue, il aspirait à faire oeuvre scientifique et neuve. Il a fait en ce sens l'effort honnête qu'on attend de tout historien. Pourtant, son oeuvre est lourde d'idéologie, d'utopie même. Seraitce qu'il n'y a pas de science innocente?

117 ASQ, Université 37, no 63, Rameau à T.-E. Hamel, 23 juin 1889. La phrase s'applique aux Canadiens, dans la lettre.

118 J.-P. Hautecoeur, L'Acadie du discours, Pour une sociologie de la culture acadienne (Québec, 1975). Cf. notre note critique dans Les Cahiers de la Société historique acadienne, 8,3 (sept. 1977): 147-157. Sur le problème de l'objectivité, c'est-à-dire de la liberté de l'historien face à sa propre idéologie, voir notre article "Â quoi servent les historiens?", L'Action nationale, 67, 6 (fév. 1978): 446-451. 